\title{
Estudio psicométrico-clínico de prevalencia y comorbilidad del trastorno por déficit de atención con hiperactividad en Castilla y León (España)
}

\author{
L. Rodríguez Molineroa , JA. López Villalobos ${ }^{b}$, M. Garrido Redondoc, \\ AM. Sacristán Martín ${ }^{d}$, MT. Martínez Rivera ${ }^{e}$, F. Ruiz Sanz ${ }^{\dagger}$ \\ ${ }^{a}$ Pediatría. CS Huerta del Rey. Valladolid. España.

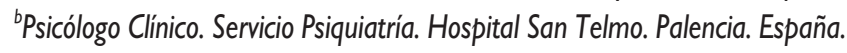 \\ PPediatría. CS la Tórtola. Valladolid. España. \\ ${ }^{` P}$ Pediatría. CS Pintor Oliva. Palencia. España. \\ ePediatría. CS Tudela de Duero. Valladolid. España. \\ fPsiquiatra. Servicio de Psiquiatría. Hospital San Telmo. Palencia. España.
}

Rev Pediatr Aten Primaria. 2009; I 1:25 1-70

Luis Rodríguez Molinero, Irmolinero@hotmail.com

\begin{abstract}
Resumen
Objetivo: determinar la tasa de prevalencia y comorbilidad del trastorno por déficit de atención con o sin hiperactividad (TDAH) en escolares de 6 a 16 años de Castilla y León (España).

Material y métodos: estudio poblacional con diseño de muestra polietápico estratificado, proporcional y por conglomerados. Población diana: 212.657. Muestra: 1.095. Se implementó una primera fase psicométrica (Attention-Deficit/Hiperactivity Disorder Rating Scales IV para padres y profesores) y una segunda fase de confirmación clínica según criterios DSM-IV. La comorbilidad se estudió mediante el Child Symptom Inventory y confirmación clínica DSM-IV.

Resultados: indican una tasa global de prevalencia del 6,66\% (IC 95\%: 5,1-8,1\%), de los cuales el 1\% fueron del subtipo hiperactivo, el 1,27\% del subtipo inatento y el 4,38\% del subtipo combinado. La tasa de prevalencia en el sexo masculino $(8,97 \%)$ es significativamente superior a la del sexo femenino (4,17\%). No aparecen diferencias significativas por cursos, ni por tipo de centro, ni por zona. La tasa de prevalencia en educación primaria $(6,8 \%)$ es similar a la de educación secundaria (6,48\%). El 71\% de los casos de TDAH presenta algún tipo de comorbilidad, con un $46 \%$ en trastornos de ansiedad, 31\% trastornos de comportamiento, $9 \%$ trastornos de estado de ánimo, 10\% trastornos por tics y $12 \%$ enuresis. Los casos de TDAH presentan significativamente peores resultados académicos y peor conducta en clase.

Conclusiones: Castilla y León tiene una prevalencia de TDAH próxima a la media mundial, cuyos casos presentan alta comorbilidad y afectación significativa en el rendimiento académico y comportamiento escolar.
\end{abstract}

Los autores declaran no presentar conflictos de intereses en relación con la preparación y publicación de este artículo. 
Palabras clave: Prevalencia, Trastorno por déficit de atención con hiperactividad (TDAH), Comorbilidad, Infancia, Adolescencia.

Abstract

Objective: to determine the prevalence rate of attention deficit hyperactivity disorder (ADHD) in school age children (6-16 years) in Castilla y León.

Subjects and methods: a multi-staged stratified proportional cluster (rural and urban) people sample population study was selected. Target population: 212,657. Sample: 1,095. A psychometric first step (ADHD rating Scales IV for parents and teachers) was used to collect data, and a second one, using DSM-IV criteria, for clinical confirmation. Child Symptom Inventory was used to study comorbidity, and DSM-IV for clinical confirmation.

Results: a prevalence of $6.66 \%$ global rate was obtained (IC $95 \%: 5.1-8.1 \%$ ). We also obtained hyperactive subtype $1 \%$; disattentional subtype $1.27 \%$ and combined subtype $4.38 \%$.

Prevalence rate in males (8.97\%) is significantly higher than in females (4.18\%), while for academic levels, schools or areas, differences are not significant. Prevalence rates in Primary School (6.8\%) is similar to Secondary School (6.48\%). Seventy one percent of the cases of ADHD have some type of comorbidity: anxiety disorders $46 \%$, behavioural disorders $31 \%$, mood disorders $9 \%$, tics disorders $10 \%$, and enuresis $12 \%$. ADHD cases have significantly worse academic results and worse classroom behaviour.

Conclusions: prevalence rates of ADHD in Castilla y León are very close to the world average. The cases have high comorbidity and present impairment in academic performance and scholar behaviour.

Key words: Prevalence, Attention-deficit/hiperactivity disorder (ADHD), Comorbidity, Childhood, Adolescence.

\section{Introducción y objetivos}

El objetivo de nuestro trabajo es estudiar la prevalencia y comorbilidad del trastorno por déficit de atención con o sin hiperactividad (TDAH) en la Comunidad Autónoma de Castilla y León (España).

En estas primeras líneas queremos dejar constancia de que el proyecto y la idea original del estudio surgen del grupo de trabajo para TDAH, auspiciado por la Asociación Española de Pediatría de Atención Primaria (AEPap).

El TDAH se caracteriza por un patrón mantenido de inatención y/o hiperactivi- dad-impulsividad, que es más frecuente y grave que el observado en sujetos de un nivel de desarrollo similar. Los síntomas, según criterios DSMIV-TR ${ }^{1}$, deben presentarse en dos o más ambientes, antes de los 7 años de edad y causar un deterioro clínicamente significativo de la actividad social, académica o laboral. El manual diagnóstico y estadístico de los trastornos mentales (DSMIV-TR), clasifica tres subtipos de TDAH, en función del patrón sintomático predominante en los últimos seis meses: Tipo combinado (TDAH-C), tipo con predominio del déficit de atención 
(TDAH-I) y tipo con predominio hiperactivo-impulsivo (TDAH-H).

El cuadro clínico analizado manifiesta un interés relevante en la actualidad tanto en el ámbito clínico como en el de las neurociencias y es un motivo frecuente, en población infantil, de remisión a neurólogos, pediatras, psiquiatras infantiles y psicólogos clínicos ${ }^{2,3}$; siendo uno de los más importantes problemas clínicos y de salud pública en términos de morbilidad y disfuncionalidad ${ }^{4}$.

La tasa de prevalencia del TDAH más citada entre los niños en edad escolar oscila entre 3 y $5 \%$ (DSM-IV) ${ }^{1}$. La bibliografía sobre estudios de prevalencia es amplia, por lo que citaremos algunos autores que realizan una revisión de estudios comunitarios y siguen criterios DSM-IV. Buitelar et al. ${ }^{5}$ citan tasas de prevalencia que oscilan entre el 4 y $17 \%$ y López-Villalobos $^{6}$ resume un amplio conjunto de estudios epidemiológicos que sitúan la prevalencia entre el $2,4 \%$ y $17,8 \%$. En esta dimensión, un estudio reciente que realizó una revisión sistemática de estudios de prevalencia mundial de TDAH (América del Norte, América del Sur, Europa, África, Asia, Oceanía y el Oriente Medio) entre los años 1978 y 2005 y en población hasta los 18 años, encontró una prevalencia media en torno al 5,29\%, observando una gran variabilidad'.
En población española podemos agrupar un conjunto de estudios con cifras de prevalencia entre el 1,2 y el $8 \%$.

La variabilidad en las cifras de prevalencia está influenciada por la determinación de la muestra, la estrategia clínica y/o psicométrica, el punto de corte utilizado en las escalas, el informante, la edad, la fuente de información, la situación geográfica, el criterio diagnóstico y la inclusión o no de deterioro en la definición ${ }^{6,7}$. Todo ello hace pensar que la comparación de las cifras obtenidas en diferentes estudios no es sencilla y debe ser realizada con precaución, pues tenemos constancia de que la utilización de muestras de mayor edad, incluir medidas de deterioro o utilizar dos informantes en vez de uno tienden a ofrecer una prevalencia mas baja, mientras que la utilización del DSM-IV frente a DSM-IIIR o CIE 10 aumenta la prevalencia ${ }^{6}$.

Las citas sobre prevalencia de subtipos de TDAH en población general y mediante la utilización del DSM-IV, suelen ofrecer resultados en los que el subtipo más frecuente es TDAH-I y secundariamente TDAH-C ${ }^{6,9}$. Esta situación normalmente se invierte en población clínica ${ }^{9}$ y recientemente sucede lo mismo en algunos estudios de prevalencia sobre TDAH, donde el subtipo TDAH-C es el más frecuente $e^{10,12}$. 
El TDAH suele presentar altas cifras de comorbilidad con trastornos del comportamiento, estado de ánimo, ansiedad y del aprendizaje ${ }^{13,15}$. Los estudios epidemiológicos de comorbilidad suelen presentar gran variabilidad en sus resultados, observando que la comorbilidad con trastornos de comportamiento se sitúa en torno al $50 \%{ }^{9,16}$; con trastornos por ansiedad se encuentran cifras que rondan el $25 \%^{17}$; con trastornos del estado de ánimo entre el $15-30 \%{ }^{13}$ y con trastornos de aprendizaje alrededor del 20$25 \%$. En el ámbito de los subtipos de TDAH (DSM-IV) sintetizamos los últimos estudios indicando que es frecuente encontrar más alteraciones del comportamiento en el fenotipo hiperactivo impulsivo (TDAH-C/TDAH-H) y ausencia de diferencias significativas entre el fenotipo referenciado previamente y el inatento (TDAH-I), en trastornos depresivos y an$\operatorname{siosos}^{18,19}$.

El objetivo principal de nuestro trabajo es determinar la prevalencia de TDAH en niños de 6-16 años en Castilla y León. Como objetivos secundarios nos interesa determinar la comorbilidad de los casos de TDAH y su distribución entre los subtipos del trastorno, así como las diferencias en rendimiento escolar entre niños con TDAH y con ausencia del problema. En el contexto de nuestros objetivos, es- tudiaremos diferencias en función de las variables sexo y edad.

La pertinencia de nuestro estudio radica en que el TDAH es uno de los más importantes problemas clínicos y de salud pública en términos de morbilidad y disfuncionalidad en la población infanto juvenil y son necesarios estudios que analicen la magnitud específica del problema y su comorbilidad, con la finalidad de que se desarrollen programas institucionales ajustados. Paralelamente, son escasos los estudios de prevalencia en nuestro país que sumen una primera fase psicométrica a una segunda clínica, garantizando una mayor precisión de los resultados. Nuestro análisis pretende colaborar en esa dimensión.

\section{Material y métodos}

\section{Población}

La población objeto de estudio estaba formada por todos los alumnos de educación primaria y secundaria desde los 6 a 16 años de la Comunidad Autónoma de Castilla y León. El número total de centros escolares en nuestra comunidad es de 1.139 (963 públicos y 276 privadosconcertados) con una población de alumnos de 212.567.

El diseño de la muestra fue polietápico, estratificado y proporcional por conglo- 
merados. La proporcionalidad respetó los bloques de educación primaria/secundaria, tipo de centro escolar (público/privado) y la zona sociodemográfica (rural y urbana). Los conglomerados se refieren al número de unidades por curso en cada centro escolar.

El cálculo del tamaño muestral se realizó según la formulación: $\mathrm{n}=\mathrm{N}{ }^{*} \mathrm{Z}_{\alpha}^{2} p^{*}$ $q / d^{2}(\mathrm{~N}-1)+\mathrm{Z}_{\alpha}^{2} * p^{*} q$. La población (N) total es 212.657 alumnos de 6 a 16 años. Se consideró un error muestral de 0,05 para una prevalencia esperada del $5 \%$ y precisión $\pm 1,4$. Nivel confianza: $95 \%$. Con estos datos, el tamaño mínimo de la muestra es de 932 alumnos (fracción de muestreo \% =0,438), ampliado a 1.200 casos en previsión de pérdidas.

La muestra recogida fue de 1.095 casos, con 661 de educación primaria y 434 de educación secundaria. La media de edad de la muestra fue 10,87 (DE [desviación estándar]: 3,05); distribuyéndose en un $51,87 \%$ sexo masculino (media edad: 10,73 ; DE: 3 ) y $48,12 \%$ femenino (media edad: 11,01; DE: 3,11).

El protocolo de nuestra investigación fue presentado y aceptado por la Comisión Regional de Salud Escolar formada por técnicos de las Consejerías Sanidad y Educación de Castilla y León. La Comisión de Ética del Área de salud Oeste de
Valladolid consideró procedente la realización del estudio. Se envió por carta a los padres de los alumnos un documento de consentimiento informado y la invitación a participar. Los padres incluidos en el estudio aceptaron y firmaron el consentimiento informado, teniendo ocasión de preguntar al investigador principal cuantas dudas tuvieran.

Los criterios de inclusión fueron: todos los alumnos de los centros y aulas seleccionados al azar que aceptaron su participación en el estudio en educación primaria y secundaria.

\section{Instrumentos}

Los padres y profesores rellenaron la Attention-Deficit/Hiperactivity Disorder Rating Scales IV (ADHD RS-IV) de DuPaul et al. ${ }^{20}$. El cuestionario usado coincide con los criterios DSM-IV y permite clasificar los subtipos de trastorno por déficit de atención con hiperactividad tipo combinado (TDAH-C), tipo con predominio de déficit de atención (TDAH-I) y tipo con predominio hiperactivo-impulsivo (TDAH-H). Cada ítem se puntúa de 0 a 3, según la respuesta dada a una escala de frecuencia que oscila entre casi nunca, algunas veces, a menudo y con mucha frecuencia. La suma de puntuaciones directas se transforma en percentiles en función de si responden profeso- 
res o padres, edad del sujeto y sexo. El manual refiere buenos indicadores de fiabilidad, validez, sensibilidad y especifidad.

Padres y profesores implementaron un apartado de la escala de evaluación Vanderbilt $\mathrm{NICHQ}^{21}$, referido a resultados académicos globales (lectura, matemáticas, expresión escrita) y conducta global en clase, susceptible de respuesta en una escala ordinal de 1 a 5 que oscila entre la consideración de resultados problemáticos, medios y superiores a la media.

Los padres de los alumnos que fueron considerados TDAH, en la primera fase de nuestro estudio, por superar el percentil 90 (PC 90) del ADHD RS-IV en padres y profesores, contestaron al Child Symptom Inventory (CSI) de Gadow y Sprafkin $^{22}$. El instrumento fue utilizado con la finalidad de analizar la comorbilidad con el TDAH e incluye un análisis de cuadros diagnósticos ajustados a criterios DSM-IV. Cada ítem es respondido por los padres según las categorías de frecuencia de nunca, a veces, a menudo y muy a menudo. El instrumento posee buena fiabilidad test-retest, muestra un alto grado de correspondencia con el diagnóstico psiquiátrico (validez predictiva), y correlaciona con otras escalas dimensionales (validez concurrente).
En función del tamaño de la muestra de TDAH y con la finalidad de realizar análisis compatibles con la literatura científica que contengan suficiente número de casos, hemos agrupado las siguientes categorías:

- Trastorno por déficit de atención con hiperactividad (categoría única).

- Trastornos del comportamiento: incluye las categorías de negativismo desafiante y trastorno disocial.

- Trastornos por ansiedad: incluye las categorías trastorno por ansiedad generalizada, fobia específica, fobia social, trastorno por ansiedad de separación, trastorno obsesivo compulsivo y trastorno por estrés postraumático.

- Trastorno del estado de ánimo (unipolar): incluye las categorías de trastorno distímico y trastorno depresivo mayor.

- Trastorno por tics: incluye tics motores $y / 0$ tics vocales.

- Enuresis (categoría única).

\section{Procedimiento}

El estudio se realizó a lo largo del curso académico 2007-2008. El diseño de la muestra permitió que se seleccionaran de forma aleatoria un total de 21 centros escolares (11 de educación primaria y 10 
de educación secundaria), y posteriormente mediante otra selección aleatoria de 33 unidades de primaria y 20 de secundaria, respetando la proporcionalidad sobre el tipo de centro y zona sociodemográfica. Si un centro se negaba a participar, se seleccionaba el siguiente del listado de centros referenciados en la Consejería de Educación con iguales características (público o privado, rural o urbano).

Se estudió el número de alumnos por unidad y se escogía otra unidad si el número no era el planificado. Se consideró que al menos debería haber 16 alumnos en educación primaria (pública) y 21 en educación primaria concertada; así como al menos 20 alumnos en educación secundaria (públicos y privados).

El trabajo de campo constaba de dos fases. La primera fase fue de cribado, se utilizaron criterios ADHD RS-IV con modelo clasificatorio similar al utilizado por Cardo et $a^{12}$. Se seleccionaron aquellos cuestionarios cuyas puntuaciones eran iguales o superiores al PC 90 según edad y sexo (baremos estadounidenses), tanto para padres como para profesores.

En esta primera fase se consideró TDAH-C cuando, tanto en cuestionario de padres como el de profesores, igualaba o superaba el PC 90 de la escala total.
Se consideró TDAH-I cuando para los dos evaluadores igualaba o superaba el PC 90 de la escala de inatención pero no en las demás; o bien cuando en uno de los evaluadores se cumple la anterior condición y para el otro se iguala o supera el punto de corte en la escala total (pero no en la de hiperactividad-impulsividad).

Se consideró TDAH-H cuando para los dos evaluadores igualaba o superaba el PC 90 de la escala de hiperactividad pero no en las demás; o bien cuando en uno de los evaluadores se cumple la anterior condición y para el otro se iguala o supera el punto de corte en la escala total (pero no en la de inatención).

Los alumnos que superan la primera fase de cribado (psicométrica) son considerados con sospecha de TDAH y son evaluados en la segunda fase (clínica).

En la segunda fase de nuestro estudio, mediante entrevista, se analizó la consistencia clínica de los casos de TDAH extraídos mediante el criterio psicométrico del ADHD RS-IV. Se dejó constancia de la existencia de todos los criterios ( $A, B, C$, $D$ y E) del DSM-IV para los casos que finalmente fueron definidos como TDAH en nuestro estudio de prevalencia. Esta valoración clínica requiere el estricto cumplimiento de los ítems necesarios para el diagnóstico y preguntas directas sobre si los síntomas se producen antes de los 7 
años de edad, están presentes en dos o más ambientes, existencia de alteración académica o social provocada por los síntomas y valoración de que los síntomas no aparecen exclusivamente en el transcurso de un trastorno generalizado del desarrollo, esquizofrenia u otro trastorno psicótico, y no se explican mejor por la presencia de otro trastorno mental.

En esta segunda fase de nuestro análisis se estudió la comorbilidad de los casos de TDAH, considerando dos criterios sucesivos. En primer lugar los padres de todos los casos de TDAH, implementaron el $\mathrm{CS}^{22}$ y con posterioridad se realizó una entrevista clínica en la que se analizaron todas las posibles comorbilidades que superasen el punto de corte categorial definido por la escala. En esta entrevista se utilizaron criterios DSM-IV para garantizar que los casos que superasen el punto de corte de una determinada comorbilidad, son realmente trastornos con la relevancia clínica que define el DSM-IV.

\section{Análisis de datos}

Se utilizaron estadísticos descriptivos y exploratorios. Siempre que fue necesario estudiar asociación o diferencias entre medidas de las diferentes variables se consideró un nivel de significación $\leqslant 0,05$. Los intervalos de confianza (IC) utilizados en las estimaciones de parámetros tendrán un nivel de confianza del 95\%.

La prevalencia fue expresada en porcentajes y se consideró el IC al 95\% para las proporciones/porcentajes siguiendo el método score, procedente del trabajo de Wilson ${ }^{23}$.

Para valorar la independencia de variables expresadas a nivel nominal se consideró la prueba de Ji cuadrado, con la corrección de Yates cuando la distribución de los datos lo aconsejaba. En algunas comparaciones de medias se consideró al análisis de varianza de un factor, implementando la corrección de Bonferroni cuando existían tres contrastes.

Para valorar diferencias de rangos promedios en muestras independientes $\mathrm{cu}$ yos datos han sido medidos en escalas ordinales se utilizó la prueba no paramétrica de Mann-Whitney.

Se utilizó un procedimiento de regresión logística, considerando conjuntamente las variables sociodemográficas correspondientes al sexo, edad, colegio público/privado y colegio rural/urbano como variables predictoras y la variable TDAH como variable criterio, valorando la relación de cada una de estas variables sociodemográficas (en presencia de las demás) con el TDAH y estudiando su influencia en la probabilidad de la presencia del trastorno. 


\section{Resultados}

La frecuencia de TDAH, correspondiente al criterio psicométrico de superar el percentil 90 en las escalas de padres 0 profesores del ADHD RS-IV, es del $25 \%$, mientras que superar simultáneamente dicho percentil en ambas escalas ofrece una tasa de sospecha para TDAH del 8,7\%. La tasa de prevalencia de TDAH de Castilla y León, correspondiente al criterio psicométrico y clínico es $6,66 \%$ (IC 95\%: 5,16-8,16).

Los casos de TDAH $(n=73)$, presentan una media de edad de 10,77 años (DE: $2,95)$ e incluyen un $69,9 \%$ del sexo masculino (media edad: 11,18; DE: 2,83 ) y $30,1 \%$ del femenino (media edad: 9,82; DE: 3,06$)$. La comparación entre colegios públicos/privados observó un 7,5\% de casos de TDAH en los públicos, frente a un $5,7 \%$ en los privados y el contraste colegios rurales/urbanos reflejó un 7,8\% de casos de TDAH en los rurales, frente a un $6,1 \%$ en los urbanos.

Mediante el procedimiento de regresión logística explicitado en el análisis de datos, observamos que únicamente la variable sexo tiene un efecto significativo positivo sobre el incremento de la probabilidad de que exista TDAH $(p=0,002)$. El sexo masculino presenta una razón de odss para TDAH 2,266 veces mayor que el sexo femenino (IC 95\%: 1,35- 3,79). La ausencia de diferencias significativas en la media de edad entre casos de TDAH y no TDAH fue contrastada me-

Tabla I. Tasas de prevalencia de TDAH por subtipos y sexo

\begin{tabular}{|c|c|c|c|c|c|c|}
\hline & \multirow[b]{2}{*}{ Negativo* } & \multicolumn{4}{|c|}{ Subtipo TDAH } & \multirow[b]{2}{*}{$\begin{array}{l}\text { TDAH } \\
\text { total }\end{array}$} \\
\hline & & Combinado & Inatento & Hiperactivo/impulsivo & Total & \\
\hline \multicolumn{7}{|l|}{ Total } \\
\hline $\mathrm{n}$ & 1022 & 48 & 14 & 11 & 1095 & 73 \\
\hline Total \% & 93,33 & 4,38 & 1,27 & 1,00 & 100 & 6,66 \\
\hline Precisión** & 1,50 & 1,25 & 1,01 & 0,87 & - & 1,50 \\
\hline \multicolumn{7}{|l|}{ Varón } \\
\hline$n$ & 517 & 35 & 10 & 6 & 568 & 51 \\
\hline Total \% & 91,02 & 6,16 & 1,76 & 1,05 & 100 & 8,97 \\
\hline Precisión** & 2,35 & 1,95 & 1,10 & 0,90 & - & 2,35 \\
\hline \multicolumn{7}{|l|}{ Mujer } \\
\hline$n$ & 505 & 13 & 4 & 5 & 527 & 22 \\
\hline Total \% & 95,82 & 2,46 & 0,75 & 0,94 & 100 & 4,17 \\
\hline Precisión b & 1,70 & 1,40 & 0,80 & 0,90 & - & 1,70 \\
\hline
\end{tabular}


diante análisis de varianza y la ausencia de asociación entre tipo de colegio o zona rural y presencia/ausencia de TDAH mediante Ji cuadrado.

La distribución de los casos de TDAH en función de los subtipos es de 65,75\% en TDAH-C, 19,17\% en TDAH-I y $15,06 \%$ en TDAH-H. La media de edad sigue la sucesión de TDAH-I [media: 11,43] > TDAH-C [media: 10,73] > TDAH-H [media: 10,09]. Mediante Anova de un factor (tipos de TDAH) no observamos diferencias significativas en media de edad $(F(2)=0,368 ; p=0,531)$. La utilización de tres contrastes y la corrección de Bonferroni tampoco observa diferencias significativas en la media de edad entre TDAH-C y TDAH-H $\left(t_{(70)}=\right.$ $0,644 ; p=0,522)$, TDAH-I y TDAH-H $\left(t_{(70)}=1,119 ; p=0,267\right)$ y TDAH-I con $\operatorname{TDAH}-\mathrm{C}\left(t_{(70)}=0,776 ; p=0,440\right)$.
La tabla I expone la tasa de prevalencia de TDAH por subtipos y sexo. La tasa de prevalencia de TDAH en los varones $(8,97 \%)$ es superior a la de las mujeres $(4,17 \%)$, observándose diferencias significativas $\left[\chi^{2}(1, n=1095)=10,14 ; p=\right.$ 0,001].

El subtipo de TDAH predominante es el combinado, con una tasa de prevalencia global de 4,38\% (IC 95\%: 3,13$5,63)$. No se observan diferencias significativas en función del sexo en la distribución de los tipos de TDAH $\left[\chi^{2}(2\right.$, $n=73)=1,45 ; p=0,483$.

La tabla II presenta las tasas de prevalencia en función de los cursos correspondientes a educación primaria y secundaria. La tasa de prevalencia en educación primaria es de 6,80\% (IC $95 \%: 4,85-8,75)$, similar a la de educación secundaria (6,48\% [IC 95\%: 4,13-

Tabla II. Tasas de prevalencia de TDAH según educación primaria o secundaria

\begin{tabular}{|c|c|c|c|c|c|c|}
\hline & \multirow[b]{2}{*}{ Negativo* } & \multicolumn{4}{|c|}{ Subtipo TDAH } & \multirow[b]{2}{*}{$\begin{array}{l}\text { TDAH } \\
\text { total *** }\end{array}$} \\
\hline & & Combinado & Inatento & Hiperactivo/impulsivo & Total & \\
\hline \multicolumn{7}{|c|}{ Educación primaria } \\
\hline$n$ & 616 & 31 & 7 & 7 & 661 & 45 \\
\hline Total \% & 93,19 & 4,68 & 1,05 & 1,05 & 100 & 6,80 \\
\hline Precisión** & 1,95 & 1,65 & 0,85 & 0,85 & - & 1,95 \\
\hline \multicolumn{7}{|c|}{ Educación secundaria } \\
\hline$n$ & 404 & 17 & 7 & 4 & 432 & 28 \\
\hline Total \% & 93,51 & 3,93 & 1,62 & 0,92 & 100 & 6,48 \\
\hline Precisión** & 2,35 & 1,85 & 1,25 & 1 & - & 2,35 \\
\hline
\end{tabular}


Tabla III. Comorbilidad en TDAH y subtipos

\begin{tabular}{lcccc}
\hline & \multicolumn{4}{c}{ Subtipo TDAH } \\
\cline { 2 - 5 } & Combinado & Inatento & Hiperactivo/impulsivo & Total \\
\hline Comorbilidad total (n) & 35 & 9 & 8 & 52 \\
Total \% & 47,94 & 12,32 & 10,95 & 71,23 \\
\hline Trastornos de comportamiento (n) & 16 & 3 & 4 & 23 \\
Total \% & 21,91 & 4,10 & 5,47 & 31,5 \\
\hline Trastornos del estado de ánimo (n) & 3 & 4 & 0 & 7 \\
Total \% & 4,10 & 5,47 & 0 & 9,58 \\
\hline Trastornos de ansiedad (n) & 25 & 5 & 4 & 34 \\
Total \% & 34,24 & 6,84 & 5,47 & 46,57 \\
\hline Tics $(\mathrm{n})$ & 5 & 2 & 1 & 8 \\
Total \% & 6,84 & 2,73 & 1,36 & 10,95 \\
\hline Enuresis $(\mathrm{n})$ & 7 & 1 & 1 & 9 \\
Total \% & 9,58 & 1,36 & 1,36 & 12,32 \\
\hline
\end{tabular}

$8,83]$ ). No se observan diferencias significativas en los casos de TDAH en función de los bloques académicos analiza$\operatorname{dos}\left[\chi^{2}(1, n=1.095)=0,53 ; p=0,817\right]$.

El subtipo de TDAH predominante es el combinado, con una tasa de prevalencia de 4,68\% (IC95\%: 3,03-6,33) en educación primaria y del 3,93\% (IC95\%: 2,08$5,78)$ en educación secundaria. La distribución de los tipos de TDAH no difieren significativamente en los bloques académicos analizados $\left[\chi^{2}(2, n=73)=0,997\right.$; $p=0,608]$.

La tabla III refleja la comorbilidad de los casos de TDAH. El 71\% presenta algún tipo de comorbilidad, siendo las más frecuentes la agrupación de los trastornos por ansiedad (46\%), trastornos del comportamiento (31\%), tras- tornos del estado de ánimo (9\%), trastornos por tics $(10 \%)$ y enuresis $(12 \%)$. El fenotipo combinado/hiperactivo-impulsivo es más frecuente que el fenotipo inatento en trastornos del comportamiento, ansiedad, enuresis y tics; sucediendo a la inversa en los trastornos del estado de ánimo.

La distribución en los tipos de TDAH no presenta diferencias significativas entre los casos que presentan comorbilidad y los que no la tienen en el apartado de comorbilidad total $\left(\chi^{2}(2, \mathrm{n}=73)=\right.$ $0,408 ; p=0,815)$. Tampoco se observan diferencias significativas entre el fenotipo inatento y el combinado/hiperactivoimpulsivo en trastornos del comportamiento $\left(\chi^{2}\right.$ corrección de Yates $(1, \mathrm{n}=$ 73) $=0,340 ; p=0,560)$, trastornos por 
ansiedad $\left(\chi^{2}\right.$ corrección de Yates $(1, \mathrm{n}=$ 73) $=0,370 ; p=0,543)$, trastornos por tics $\left(\chi^{2}\right.$ corrección de Yates $(1, n=73)=$ $0,006 ; p=0,970)$ y enuresis $\left(\chi^{2}\right.$ corrección de Yates $(1, n=73)=0,042 ; p=$ $0,838)$. Sin embargo, sí se observan diferencias significativas en los trastornos del estado de ánimo con más frecuencia del fenotipo inatento, respecto al combinado/hiperactivo-impulsivo ( $\chi^{2}$ corrección de Yates $(1, n=73)=4,745 ; p=0,029)$.

La distribución del sexo en función de la comorbilidad de los casos de TDAH ofrece los siguientes resultados:

- Trastornos del comportamiento: suceden en el $27,5 \%(n=14)$ del sexo masculino y en el $40,9 \%$ ( $n=$ 9) del femenino. No se observan diferencias significativas $\left(\chi^{2}(1, n=\right.$ 73) $=1,290 ; p=0,256)$.

- Trastornos por ansiedad: suceden en el $43,1 \%(n=22)$ del sexo masculino y en el $54,5 \%(n=12)$ del femenino. No se observan diferencias significativas $\left(\chi^{2}(1, n=73)=\right.$ $0,804 ; p=0,370)$.

- Trastornos del estado de ánimo: suceden en el 7,8\% $(n=4)$ del sexo masculino y en el $13,6 \%(n=3)$ del femenino. No se observan diferencias significativas $\left(\chi^{2}\right.$ corrección de Yates $(1, n=73)=0,114 ; p=$ $0,735)$.
- Comorbilidad total: sucede en el $68,6 \%(n=35)$ del sexo masculino $y$ en el $77,3 \%(n=17)$ del femenino. No se observan diferencias significativas $\left(\chi^{2}(1, n=73)=0,561 ; p\right.$ $=0,454)$.

La distribución de los bloques académicos en función de la comorbilidad de los casos de TDAH ofrece los siguientes resultados:

- Trastornos del comportamiento: suceden en el $33,3 \%(n=15)$ de educación primaria y en el $28,6 \%$ $(n=8)$ de educación secundaria. No se observan diferencias significativas $\left(\chi^{2}(1, n=73)=0,181 ; p=\right.$ $0,670)$.

- Trastornos por ansiedad: suceden en el $55,6 \%(n=25)$ de educación primaria y en el $32,1 \%(n=9)$ de educación secundaria. Se observan diferencias significativas $\left(\chi^{2}(1, n=\right.$ 73) $=3,802 ; p=0,05)$, existiendo una mayor proporción en primaria.

- Trastornos del estado de ánimo: suceden en el 4,4\% ( $n=2)$ de educación primaria y en el 17,9\% ( $n=5)$ de educación secundaria. No se observan diferencias significativas $\left(\chi^{2}\right.$ corrección de Yates $(1, n=73)=$ 2,$202 ; p=0,138$ ).

- Comorbilidad total: suceden en el $75,6 \%(n=34)$ de educación pri- 
maria y en el $64,3 \%(n=18)$ de educación secundaria. No se observan diferencias significativas $\left(\chi^{2}(1\right.$, $n=73)=1,070 ; p=0,301$ ).

La utilización de la escala de evaluación Vanderbilt contestada por los profesores, refleja diferencias significativas en rangos promedios (prueba $U$ de Mann-Whitney) en las que los casos de TDAH, respecto a los que no tienen esa condición, tienen peores resultados académicos globales ( $U$ $=18852 ; p=0,000)$ e inferiores resultados en lectura $(U=22834 ; p=0,000)$, matemáticas $(U=19730 ; p=0,000)$ y expresión escrita $(U=19298 ; p=0,000)$.

El mismo cuestionario refleja que los casos de TDAH tienen peor conducta global en la clase $(U=16227 ; p=0,000)$; así como peor relación con los compañe$\operatorname{ros}(U=17653 ; p=0,000)$, menor respeto a las normas y reglas $(U=16580 ; p=$ $0,000)$, mayores interrupciones de la clase $(U=20576 ; p=0,000)$, menores destrezas de organización $(U=15706 ; p=$ $0,000)$ y conducta mas problemática para hacer los deberes $(U=16469 ; p=0,000)$.

\section{Discusión y conclusiones}

La utilización de un punto de corte en el percentil 90 (PC 90) del ADHD RS-IV y la consideración de este límite en la escala de padres o la de profesores (criterio "o") refleja una frecuencia de TDAH del
$25 \%$, mientras que utilizando el mismo procedimiento en la escala de padres y profesores (criterio " $y$ ") observamos una tasa de sospecha de TDAH del 8,7\%. Si a este criterio psicométrico le sumamos la dimensión clínica que presenta nuestro estudio, la cifra se reduce a un $6,66 \%$. Todo ello nos hace reflexionar sobre la relevancia que el método tiene en los resultados de la prevalencia y sobre la importancia de una adecuada exploración de los casos de TDAH, que no debe estar limitada a la respuesta a los cuestionarios existentes en la materia.

La tasa de prevalencia de TDAH en Castilla y León (España) es de 6,66\% (IC 95\%: 5,16-8,16), compatible en su límite inferior con el intervalo más aceptado en la comunidad científica que se sitúa entre el 3 y el $5 \%$ (DSM-IV) y con estudios que realizan una revisión sistemática de la prevalencia mundial en TDAH con una prevalencia media del $5,29 \%$. La cifra se encuentra en el intervalo de los estudios agrupados españoles y foráneos que hemos citado en la introducción ${ }^{5,6,8}$, con idénticas explicaciones sobre su variabilidad ${ }^{6,724}$.

Estudios españoles encuentran tasas de prevalencia que se sitúan entre el 1,2\% y $8 \%{ }^{8}$, entre los que podemos destacar un riguroso estudio realizado en Sevilla con una muestra de edades entre 6 y 15 años. 
Se exigió que coincidieran información escolar de profesores, criterios DSM y entrevista clínica y se obtuvo una cifra de prevalencia del $6 \%$, similar a la obtenida en nuestra investigación ${ }^{25}$. Recientemente un excelente estudio realizado en Palma de Mallorca compatible con nuestra primera fase psicométrica, encuentra una tasa de prevalencia global en TDAH del 4,57\% (IC 95\%: 3,18-5,96), inferior a la ofrecida en nuestros datos ${ }^{12}$.

La tasa de prevalencia de TDAH en los varones de nuestro estudio es significativamente superior a la de las mujeres, con una proporción de 2:1. Este resultado es habitual en la bibliografía y se encuentra citado en la DSM-IV con una proporción de 4:1 para la población general. Las proporciones observadas en población no clínica suelen ser favorables al sexo masculino con cifras de proporciones variadas. Entre ellas podemos citar algunas similares a la nuestra de 2:126 ó 2,7:127 y otras que se mueven en un margen mayor de $3: 1^{28}, 3,8: 1^{11}$ ó $5: 1^{29}$. Existe una amplia discusión acerca de estas diferencias, argumentando que si las normas de aplicación de test sobre TDAH se utilizasen considerando datos normativos estadísticos propios de cada sexo, con sus puntos de corte respectivos, las distancias disminuirían o desaparecían. Nuestro estudio no avala esta referencia, que sin embargo se confirma en otro estudio similar a la primera fase del nuestro, en alumnos entre primero y cuarto de primaria $^{12}$.

El subtipo de TDAH predominante en nuestro estudio es el combinado, con una tasa de prevalencia global de 4,38\% (IC 95\%: 3,13-5,63). Este dato tiene especial relevancia por la posibilidad de que en el futuro DSM-V el TDAH se reduzca a esta dimensión.

El subtipo inatento de TDAH refleja una prevalencia de 1,27 y en el hiperactivo-impulsivo de 1.

Las referencias de citas sobre prevalencia, utilizando criterios DSM-IV, en población general ofrecen resultados en los que el subtipo más frecuente suele ser el inatento y secundariamente el combinado; mientras que esta situación, normalmente, se invierte en población clínica ${ }^{6,9,18}$. Nuestros resultados no se mueven en esta dirección y la mayor prevalencia del tipo de TDAH-C puede deberse a la sucesión en doble fase (psicométrica y clínica) de nuestro estudio. Existen estudios epidemiológicos recientes que mediante procedimientos diferentes observan resultados similares al nuestro $^{10,11}$ y un estudio español que utiliza criterios psicométricos semejantes a nuestra primera fase ofrece resultados equivalentes ${ }^{12}$. 
La tasa de prevalencia de nuestro análisis decrece de forma leve y no significativa al comparar la educación primaria $(6,80 \%)$ y secundaria $(6,48 \%)$, no ofreciendo diferencias significativas en la distribución de los tipos de TDAH en el tránsito entre los bloques de edad analizados. Este dato apoya las referencias sobre la persistencia del trastorno en los márgenes de edad analizados ${ }^{30}$.

La prevalencia de los tipos de TDAH sigue la secuencia de TDAH-C > TDAHI > TDAH-H y se observa un decremento de la prevalencia en TDAH-C/TDAH$\mathrm{H}$ y un incremento de TDAH-I al comparar los bloques educativos analizados. Estos datos apoyan la idea de una disminución de síntomas asociados al exceso de inquietud con el incremento de la edad ${ }^{31}$.

Nuestro estudio también observa que la media de edad de los casos de TDAH sigue la secuencia TDAH-I > TDAH-C > TDAH-H. Este resultado es compatible con múltiples investigaciones que, con distintas explicaciones teóricas, dejan constancia sobre el hecho de que los síntomas de inatención tienden a persistir más en el tiempo ${ }^{18}$.

La comorbilidad reflejada en nuestro estudio alcanza al $71 \%$ de los casos de TDAH, con una media de 1,27 diagnósticos comórbidos por caso. Esta condición es frecuente en el TDAH y está probablemente asociada a que los síntomas del trastorno favorecen la posibilidad de otras alteraciones psicopatológicas, quizás como un espectro continuo de síntomas del mismo trastorno de fondo.

Los resultados de nuestro estudio presentan una comorbilidad inferior a la de algunos estudios de muestras clínicas que citan un $80-83 \%$ con un promedio de 1,4/2 diagnósticos adicionales ${ }^{32,33}$. Nuestros datos de comorbilidad son superiores a un estudio comunitario que encuentra que al menos un $44 \%$ de los casos de TDAH tienen un diagnóstico asociado $^{34}$ y sin embargo son algo inferiores al estudio realizado en Suecia en el que se observó que el $87 \%$ de niños con comorbilidad $^{35}$.

Los diagnósticos comórbidos reflejados en nuestro estudio son similares a los encontrados en la bibliografía científica, donde es frecuente la asociación con trastornos del comportamiento, ansiedad, estado de ánimo, enuresis o tics.

Los trastornos por ansiedad son el diagnóstico comórbido más frecuente, incluyendo un $46 \%$ de los casos de TDAH. Esta comorbilidad es mayor que la citada habitualmente en estudios epidemiológicos que se sitúa en torno al $25 \%{ }^{17}$ y se asemeja más a la observada en entornos clínicos que oscila entre $30-40 \%$. 
Los trastornos del comportamiento son una patología comórbida en el $31 \%$ de los casos de TDAH de nuestra muestra. Diversos estudios epidemiológicos citan cifras en torno al 50\% y los estudios con muestras clínicas suelen reflejar un incremento de esta comorbilidad respecto a los elaborados con población general ${ }^{16,36}$. La comorbilidad con los trastornos de comportamiento es tan frecuente que en ocasiones es difícil determinar si no se trata de una manifestación del propio TDAH $^{37}$.

Los trastornos del estado de ánimo unipolares representan el 9,58\% de la comorbilidad de nuestros casos de TDAH. Los estudios epidemiológicos de comorbilidad con este trastorno presentan gran variabilidad, entre el $15-75 \%{ }^{37}$, estimándose un promedio entre el 15-30\% ${ }^{13,39}$.

La comorbilidad encontrada en nuestro estudio con trastornos del comportamiento, ansiedad y del estado de ánimo adquiere su sentido e importancia cuando conocemos que la frecuencia con que cada uno de ellos aparece conjuntamente con el TDAH es superior a su presencia aislada en la población general. Estudios epidemiológicos foráneos en población infanto juvenil en el margen de edad de nuestro estudio, reflejan tasas de prevalencia en torno al $10 \%$ en ansiedad ${ }^{9}$, entre el 2 y $16 \%$ en trastorno negativista desafiante 1 , entre $6-16 \%$ en sexo masculino del trastorno disocial', entre el 2 y $9 \%$ en sexo femenino de trastorno disocial $^{1}$ y entre el 2,5 y $5,9 \%$ en trastornos depresivos ${ }^{40}$.

En síntesis, la comorbilidad encontrada en nuestro estudio epidemiológico tiene una importante magnitud y es inferior al promedio observado en diversos estudios epidemiológicos en trastornos del comportamiento y alteraciones del estado de ánimo; siendo más elevada a la habitualmente observada en trastornos por ansiedad.

En nuestro análisis el fenotipo combinado/hiperactivo-impulsivo es más frecuente que el fenotipo inatento en trastornos del comportamiento, ansiedad, enuresis y tics; sucediendo a la inversa en los trastornos del estado de ánimo. Sin embargo, solo encontramos diferencias significativas en este último aspecto. Las referencias de diversas investigaciones que utilizan criterios DSM-IV con muestras clínicas y/o escolares, encuentran más trastornos de conducta en el fenotipo combinado respecto al inatento; observando ausencia de diferencias relevantes en trastornos internalizantes ${ }^{18,20}$. También existen citas de investigaciones que encuentran mayor frecuencia de trastornos depresivos en el fenotipo inatento ${ }^{18}$. Nuestro análisis se mueve en 
consonancia con un grupo importante de los estudios referenciados.

En el capítulo de las diferencias en función del sexo en el área de la comorbilidad, observamos una mayor proporción de trastornos del comportamiento, ansiedad y del estado de ánimo en el sexo femenino, no encontrando diferencias significativas. Las investigaciones suelen citar que los varones con TDAH, suelen tener mayor asociación con los trastornos del comportamiento y las chicas con trastornos de estado de ánimo y de ansiedad, observándose algunos estudios que no encuentran diferencias de género en esta última dimensión 6 .

Si comparamos las proporciones de comorbilidad en educación primaria y secundaria, apenas se observan diferencias en trastornos del comportamiento, mientras en los trastornos por ansiedad existe una mayor proporción en educación primaria que en secundaria, sucediendo a la inversa en los trastornos del estado de ánimo. Únicamente se observan diferencias significativas en los trastornos por ansiedad que son el trastorno psicológico más frecuente en edad infantil, aunque su grado de solapamiento con TDAH es mucho mayor de lo que se esperaría por azar ${ }^{37}$.

Finalmente, como suele reflejar la bibliografía científica ${ }^{6,9,13}$, los casos de TDAH tienen significativamente peor rendimiento académico (lectura, matemáticas, expresión escrita) y conducta escolar (relación con compañeros, respeto a las normas, interrupciones de la clase, destrezas de organización, hacer los deberes) que los que no tienen ese trastorno.

En síntesis, el TDAH en la Comunidad de Castilla y León presenta una tasa de prevalencia que se sitúa discretamente por encima de los límites habituales cuando se utilizan criterios DSM-IV, tiene una alta comorbilidad principalmente con trastornos del comportamiento/ansiedad y refleja afectación significativa en el rendimiento académico y conducta escolar.

\section{Fuente de financiación}

El estudio ha contado con una ayuda a la investigación de la Fundación de Pediatría Ernesto Sánchez Villares. 


\section{Bibliografía}

1. American Psychiatric Association. Manual diagnóstico y estadístico de los trastornos mentales. Barcelona: Masson; 2002.

2. Kutcher S, Aman M, Brooks SJ, Buitelaar J, Van Daalen E, Fegert J, et al. Declaración de consenso internacional sobre el trastorno de déficit de atención e hiperactividad y los trastornos de conducta disruptiva: implicaciones clínicas y sugerencias de tratamiento. Eur Neuropsychopharmacol. 2004;14(1):11-28.

3. López JA, Serrano I, Delgado J, Ruiz F, García MJ, Sánchez MI. Trastorno por déficit de atención con hiperactividad: una alteración psicopatológica con impacto multidimensional. An Psiquiatr. 2004; 20(5):205-10.

4. Lerner M. Office-based management of ADHD. In: Satellite Symposium to the American Academy of Pediatrics National Conference and Exhibition. Boston, Massachusetts: AAP; 2002.

5. Buitelaar JK, Van Engeland H. Epidemiological approaches. In Sandberg S, ed. Hyperactivity disorders of childhood. Cambridge: Cambridge University Press; 1996. p. 26-68.

6. López Villalobos JA. Perfil evolutivo, cognitivo, clínico y socio-demográfico de las personas con trastorno por déficit de atención con hiperactividad, atendidas en una unidad de salud mental [tesis doctoral]. Universidad de Salamanca; 2002.

7. Polanczyk G, de Lima MS, Horta BL, Biederman J, Rohde LA. The worldwide prevalence of ADHD: a systematic review and metaregression analysis. Am J Psychiatr. 2007;164(6):942-8.

8. Pedreira JL. Diagnosticando el niño con hiperactividad. Psiquiatría.com. Interpsiquis, 2004 [consultado el 08/04/2009]. Disponible en http:// www.psiquiatria.com/articulos/trastornos_infan tiles/13485/
9. Brown ET. Attention - deficit disorders and comorbidities in children, adolescents, and adults. Washington DC: American psychiatric Press; 2000.

10. Montiel-Nava C, Peña JA, López M, Salas $M$, Zurga JR, Montiel-Barbero I, et al. Estimaciones de la prevalencia del trastorno por déficit de atención-hiperactividad en niños marabinos. Rev Neurol. 2002;35:1019-24.

11. Cornejo JW, Osío O, Sánchez Y, Carrizosa J, Sánchez $\mathrm{G}$, Grisales $\mathrm{H}$, et al. Prevalencia del trastorno por déficit de atención-hiperactividad en niños y adolescentes colombianos. Rev Neurol. 2005; $40: 716-22$

12. Cardo E, Servera M, Llobera J. Estimación de la prevalencia del trastorno por déficit de atención e hiperactividad en población normal de la isla de Mallorca. Rev Neurol. 2007;44:10-4.

13. Barkley RA. Attention Deficit Hyperactivity Disorder. A handbook for diagnosis and treatment. New York: Guilford Press; 2006.

14. Blázquez-Almería $G$, Joseph-Munné D, Burón-Masó $\mathrm{E}$, Carrillo-González $\mathrm{C}$, Joseph -Munné $M$, Cuyàs-Reguera $M$, y cols. Resultados del cribado de la sintomatología del trastorno por déficit de atención con o sin hiperactividad en el ámbito escolar mediante la escala EDAH. Rev Neurol. 2005; 41:586-90.

15. Miranda-Casas A, García-Castellar R, Meliade Alba A, Marco-Taverner R. Aportaciones al conocimiento del trastorno por déficit de atención con hiperactividad. Desde la investigación a la práctica. Rev Neurol. 2004;38:156-63.

16. Manuzza S, Klein RG, Abikoff H. Significance of childhood conduct problems to later development of conduct disorders among children with ADHD: a prospective follow-up study. J Abnorm Child Psychol. 2004;32:565-73.

17. Schatz DB, Rostain AL. ADHD with comorbid anxiety: a review of the current literatura. J Atten Disord. 2006;10:141-9. 
18. López JA, Montes JM, Sánchez MI. Trastorno por déficit de atención con hiperactividad: análisis discriminante de subtipos. Rev Psiquiatr Infanto-Juv. 2003;20:108-19.

19. López-Villalobos JA, Serrano I, Delgado J. Trastorno por déficit de atención con hiperactividad: comorbilidad con trastornos depresivos y de ansiedad. Psicothema. 2004;16:402-7.

20. DuPaul G, Power JT, Anastopoulos AD, Reid R. ADHD-Rating Scales DSM-IV for parents and teachers. New York: Guilford Press; 1998.

21. Wolraich ML, Lambert EW, Doffing MA, Bickman L, Simmons T, Worley K. Psychometric properties of the Vanderbilt ADHD diagnostic parent rating scale in a referred population. J Pediatr Psychol. 2003;28:559-68.

22. Gadow KD, Sprafkin J. Child Symptom Inventory 4, Norms manual. Stony Brook, NY: Checkmate Plus; 1997.

23. Newcombe RG, Merino C. Intervalos de confianza para las estimaciones de proporciones y las diferencias entre ellas. Interdisciplinaria. 2006; 23;141-54.

24. Narbona J. Alta prevalencia del TDAH: ¿niños trastornados, o social maltrecha? Rev Neurol. 2001;32:229-33.

25. Benjumea $\mathrm{P}$, Mojarro MD. Trastornos hipercinéticos: estudio epidemiológico en doble fase de una población Sevillana. An Psiquiatr. 1993;9:30611.

26. Kroes M, Kalff AC, Kessels AGH, Steyaert J, Feron FJM, Van Someten A, et al. Child psychiatric diagnoses in a population of Dutch schoolchildren aged 6 to 8 years. J Am Acad Child Adolesc Psychiatry. 2001;40:1401-9.

27. Rowland A, Umbach DM, Catoe KE, Stallone L, Long S, Rabiner D, et al. Studying the epidemiology of attention-deficit hyperactivity disorder: Screening method and pilot results. Can J Psychiatry. 2001;46:931-40.
28. Costello EJ, Angold A, Burns BJ. The Great Smoky Mountains study of youth: coals, design, methods, and the prevalence of DSM III R disorders. Arch Gen Psychiatry. 1996;53:1129-36.

29. Gomez R, Harvey J, Quick C, Scharer I, Harris G. DSM-IV AD/HD: confirmatory factor models, prevalence, and gender and age differences based on parent and teacher ratings of Australian primary school children. J Child Psychol Psychiatry. 1999;40:265-74.

30. Mulas F, Etchepareborda MC, Abad-Mas L, Díaz-Lucero A, Hernández $S$, de la Osa $A$, y cols. Trastornos neuropsicológicos de los adolescentes afectos de trastorno por déficit de atención con hiperactividad. Rev Neurol. 2006;43 (Supl 1):s71s81.

31. Biederman J, Mick E, Faraone S. Age-dependent decline of symptoms of attention deficit hyperactivity disorder: impact of remission definition and symptom type. Am J Psychiatry. 2000;157: 816-8.

32. Wilens TE, Biederman J, Brown S, Tanguay S, Monuteaux MC, Blake C, et al. Psychiatric comorbidity and functioning in clinically referred preschool children and school-age youths with ADHD. J Am Acad Child Adolesc Psychiatry. 2002; 41:262-8.

33. López-Villalobos JA, Rodríguez $L$, Sacristán A, Garrido M, Martínez T. El psicólogo clínico y el trastorno por déficit de atención con hiperactividad: vinculación con el pediatra. Rev Pediatr Aten Primaria. 2006;8:157-73.

34. Szatmari P, Offord DR, Boyle MH. Ontario Child Health Study: prevalence of attention deficit disorder with hyperactivity. J Child Psychol Psychiatry. 1989;30:219-30.

35. Kadesjo B, Gillberg C. The comorbidity of ADHD in the general population of Swedish school-age children. J Child Psychol Psychiatr. 2001;42: 487-92. 
36. López-Villalobos JA, Serrano I, Delgado J. Attention Deficit Hyperactivity Disorder: a predictive model of comorbidity with behaviour disorder. Psychology in Spain. 2005;9:63-74.

37. Artigas J. Comorbilidad del trastorno por déficit de atención e hiperactividad. Rev Neurol. 2003;36 (Supl 1):S68-78.

38. Osa-Langreo A, Mulas F, Téllez de Meneses M, Gandía R, Mattos L. Psicofarmacología de los trastornos comórbidos asociados al trastorno por déficit de atención/hiperactividad. Rev Neurol. 2007;44 (Supl 2):s31-s35.
39. López-Villalobos JA, Ruiz F. Dimensiones depresivas en el trastorno por déficit de atención con hiperactividad. An Psiquiatría. 2006;22: 271 81.

40. Méndez FX, Olivares J, Ros MC. Características clínicas y tratamiento de la depresión en la infancia y adolescencia. En: Caballo VE, Simón MA, dir. Manual de psicología clínica infantil y del adolescente. Madrid: Pirámide; 2005. p. 139-85. 